\title{
Photoelectrical Behaviour of DNA:PEDT-PSS Functionalised Films
}

\author{
V. KaŽUkauskas ${ }^{a, *}$, M. Pranaitis $^{a}$, O. Krupka $^{b}$ And B. Sahraoui ${ }^{b}$ \\ ${ }^{a}$ Semiconductor Physics Department and Institute of Applied Research, Vilnius University \\ Saulètekio al. 9, bldg. 3, LT-10222 Vilnius, Lithuania \\ ${ }^{b}$ MOLTECH Anjou - UMR CNRS 6200 MINOS, University of Angers \\ 2 Blvd. Lavoisier, 49045 Angers, France
}

\begin{abstract}
We report investigations of functionalized DNA:PEDT-PSS films. The thermal activation energy of the conductivity near the room temperature was about $0.033 \mathrm{eV}$. The weak carrier trapping was identified by the thermally stimulated current method, proving the fast recombination of light-generated carriers. A "bistable" photoconductivity below the room temperature was evidenced upon the white light excitation. By cooling the samples down to 145-155 K the photoconduction was small. Below this temperature sudden increase of the photoconductivity was observed. Meanwhile by heating the photosensitivity remained enhanced up to $235-245$ K. The slow relaxations of the current after the light excitation took place, the time constant of which reached several hundreds of seconds. Such phenomenon could presumably be attributed to the light-induced changes of the sample material morphology and/or associated variation of carrier transport conditions.
\end{abstract}

PACS: 73.50.Gr, 81.40.Rs, 81.40.Tv, 87.85.jc, 87.14.gk

\section{Introduction}

Nucleic acids and their derivatives attract a lot of attention due to their important biological role $[1,2]$. At the same time deoxyribonucleic acid (DNA), in which charge migration is possible, is a subject of interest for applications in photonics and molecular electronics. However, pure DNA is electrically passive material. In order to render it active one has to functionalize it with poly(3,4-ethylenedioxythiophene) (PEDOT, PEDT) and poly(styrenesulfonate) (PSS) complex. PEDT-PSS is a promising polymer for electronic applications because it is solution processable. When dried, PEDT-PSS coatings have low resistivity, excellent water resistance, and high transmittance of visible light. This enables engineering of the electrical properties of the material. Nevertheless, to assure the control of these properties, extensive investigations are required. In this paper we report investigations of photoelectrical properties of DNA:PEDTPSS thin films depending on the temperature and light excitation.

\section{Samples and experiment}

PEDT-PSS (Fig. 1) aqueous dispersion is available as the trade mark of BAYTRON ${ }^{\circledR} \mathrm{PH} 500$. The weight ratio of PEDT to PSS is about 1:2.5. DNA sodium salts with molecular weight $M_{\mathrm{W}}=10^{6}$ Da, extracted from

* corresponding author; e-mail: vaidotas.kazukauskas@ff.vu.lt salmon milt and roe, were provided by CIST. After sonication of DNA $(8 \mathrm{~g} / \mathrm{L})$ in deionized water, the DNA solution was added to PEDT-PSS aqueous solution with volume ratios 1:0.2 and 1:0.5. The solutions were spin-coated on the ITO glass substrates. The obtained films of $0.3-1.5 \mu \mathrm{m}$ thick were homogeneously intermixed to molecular level. The semi-transparent aluminium contacts were evaporated on the top. The samples measured about $5 \times 5 \mathrm{~mm}^{2}$. The temperature dependences of the film conductivity were measured from $77 \mathrm{~K}$ up to $300 \mathrm{~K}$ depending on the excitation by the white light from the $100 \mathrm{~W}$ halogen lamp.

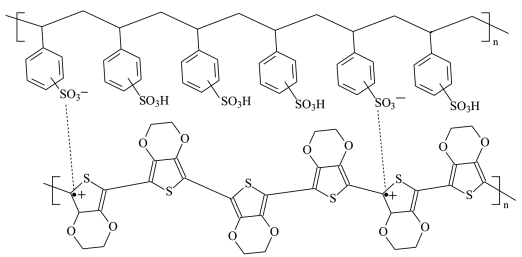

Fig. 1. Chemical structure of polymer complex PEDT-PSS

\section{Results and discussion}

Sample conductivity at the room temperature was $(1-5) \times 10^{-10} \Omega^{-1} \mathrm{~cm}^{-1}$. $I-V$ curves were linear and symmetrical down to $78 \mathrm{~K}[3]$. This ohmic behaviour indicates that the current is limited by the sample material and not by the contacts. Absorption spectra of the samples are presented in [3]. In Fig. 2 temperature de- 
pendences of the currents are shown. Close to the room temperature their thermal activation energy was about $0.033-0.036 \mathrm{eV}$; these values did not depend on the applied bias. Usually thermal activation energy values of DNA from 0.18 up to $1.4 \mathrm{eV}$ are reported, depending on material and experimental conditions [4]. This implies that in our samples influence of PEDOT having high conductivity is pronounced. Below about $130-140 \mathrm{~K}$ a characteristic kink appears, and the thermal activation energy drops below $0.014 \mathrm{eV}$, approaching in some samples zero values. The similar trend was observed in DNA-gold composite [5] because of the change of the charge transport mechanism from the small polaron hopping at higher temperatures $[6,7]$ to the Mott variable-range hopping $[7,8]$ at low temperatures.

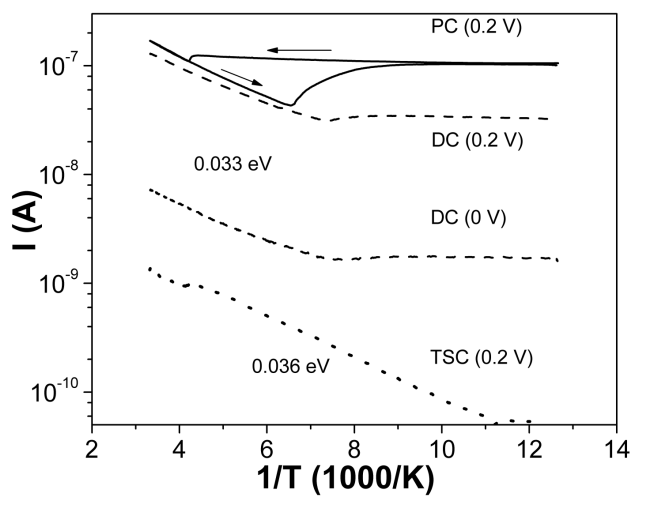

Fig. 2. Temperature dependences of the photocurrent (solid curves), upon scanning the temperature down and up as indicated by the arrows, the dark current (dashed curves) at $0.2 \mathrm{~V}$ bias and without any applied bias and TSC (dotted line).

To analyse the possible effect of carrier trapping, the thermally stimulated currents (TSC) were investigated as described in $[9,10]$. Characteristically, TSCs, even after the full saturation of the current of the illuminated samples, remained low as compared with both dark and photocurrent (Fig. 2). This implies that relatively small number of carriers remain trapped after the light is switched off, i.e., most of them recombine after the excitation is finished. Moreover, nearly the same activation energy values of about $0.036-0.043 \mathrm{eV}$ were evaluated down to the lowest temperatures. This indicates that carrier trapping conditions remain unchanged in the whole temperature region, meanwhile properties of the conductivity change notably. On the other hand, such low activation energy values could be indication that current increase with temperature is related to transport phenomena, i.e., growth of carrier mobility, rather than change of the carrier density. Similar behaviour is reported in disordered organic solar cell structures [11]. This assumption is realistic by having in mind hopping transport character in disordered organic materials. Therefore evaluated activation energies could probably reflect the energetic spread in the charge transport states.
The samples had demonstrated photosensitivity starting from the room temperature down to $78 \mathrm{~K}$ (Fig. 2). At the room temperature the current used to grow by $\approx 2-3 \%$, and at LN temperature its growth by a factor of up to two was observed. Sample contacts remained ohmic in all cases, indicating that carriers were photogenerated in the sample material. Dependence of the photocurrent on the temperature was quite complex. A characteristic kink, which was observed in the dark current measurements, becomes more expressed upon light excitation. By lowering the temperature the photocurrent starts growing at $145-155 \mathrm{~K}$ and reaches saturation level that is by a factor of about 2-2.5 higher than that of the minimum. Afterwards, by increasing the temperature, the photocurrent does not change up to 235-245 K, forming a "bistable" loop. This behaviour does not depend on the bias, indicating that the effect is light-induced. This is supported by the fact that sample reaction on the voltage and light pulses was different: upon application of a voltage step very fast increase of the current was observed, meanwhile the photocurrent growth was slow [3]. This evidences effect of different mechanisms. Upon application of the voltage pulse fast carrier injection from contacts takes place that is limited by ohmic conduction of the sample volume. Meanwhile slower light-induced generation of carriers occurs from their transport and/or trapping states.

The behaviour of the current by scanning the temperature upon pulsed illumination of the samples is presented in Fig. 3. The temperature was stabilized at $210 \mathrm{~K}, 175 \mathrm{~K}$, i.e., within the bistable loop, and at $80 \mathrm{~K}$. At these points the sample was excited by the light pulses. In Fig. 4 behaviour of the relative photoconductivity $\frac{I_{\text {light }}-I_{\text {dark }}}{I_{\text {dark }}}$ is presented. Upon cooling down to the kink point, the photosensitivity remains low. At the temperatures below this point the photocurrent increases significantly, moreover, its maximum reaches the same value upon heating the sample (Fig. 3). Meanwhile the relative photoconductivity reaches $\approx 0.7$ at $80 \mathrm{~K}$ (Fig. 4). The photocurrent upon illumination increases much faster than it drops after the light is turned off, though both processes could be approximated by the single exponents. This indicates the complex effect that cannot be attributed to the simple model, e.g., carrier generation from single trapping centres. The long relaxation "tails" indicate appearance of the persistent photoconductivity phenomenon [12]. This phenomenon is associated with the inability of the light-generated carriers to recombine, as they become trapped in the spatially separated potential wells and hills of the band gap edges in the disordered structure. Upon heating the sample relaxation times used to shorten as indicated in Fig. 4. Characteristically, amplitudes of the relative photoconductivity had followed qualitatively similar behaviour.

Therefore the bistability of photoconductivity could probably be attributed to the light-induced morphology changes of the samples, resulting in an increase of photosensitivity at low temperatures. To explain this effect 


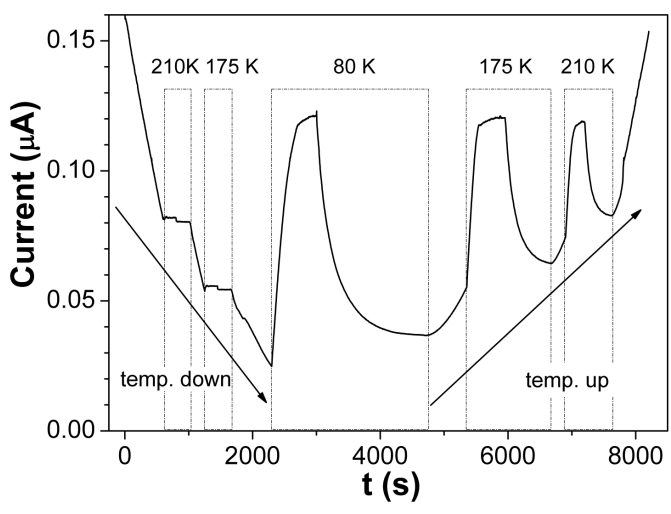

Fig. 3. Change of the current upon variation of the temperature and pulsed illumination of the sample. The temperature was stabilized at $210 \mathrm{~K}, 175 \mathrm{~K}$, and at $80 \mathrm{~K}$, as indicated by the dashed-dotted rectangles. At these points the sample was excited by the white light pulses.

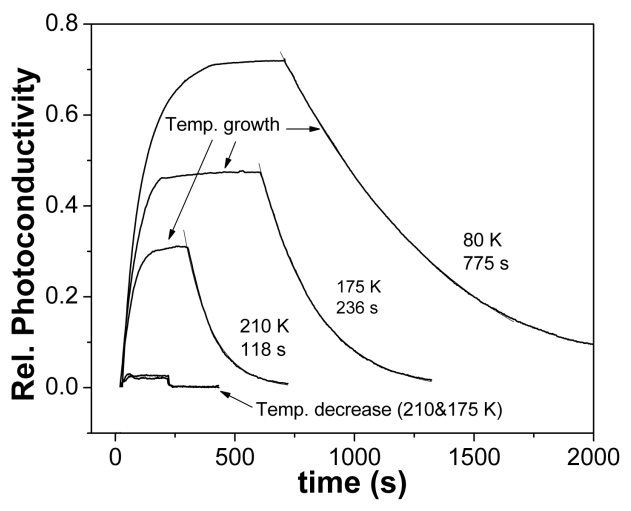

Fig. 4. Kinetics of the relative photoconductivity upon excitation of the sample by the white light pulses at different temperatures by cooling and heating.

several mechanisms can be accounted for. First of all, it is known that an anisotropic conductivity is characteristic for PEDT-PSS thin films [13]. So, one can suppose that light excitation could energize molecular movements of PEDT-PSS, changing in this way the predominant orientation of PSS lamellas. The light induced variation of PEDOT conjugation lengths was reported in [14]. Another possible mechanism could be variation of the transition temperature at which change of the transport mech- anism occurs. This could be assisted by the light-induced variation of the density of hopping states, because of their filling by generated carriers.

\section{Acknowledgments}

This work was supported by the Lithuanian Ministry of Education and Science and EGIDE Agency (France) within Gilibert program and by the Research Council of Lithuania (projects No. TAP-15 and TAP-64).

\section{References}

[1] X. Dou, T. Takama, Y. Yamaguchi, K. Hirai, H. Yamamoto, S. Doi, Y. Ozaki, Appl. Opt. 37, 759 (1998).

[2] S. Asayama, A. Maruyama, Ch.-S. Cho, T. Akaike, Bioconjugate Chem. 8, 833 (1997).

[3] V. Kažukauskas, A. Arlauskas, M. Pranaitis, O. Krupka, F. Kajzar, Z. Essaidi, B. Sahraoui, Opt. Mater. 32, 1629 (2010).

[4] E.P. Bellido, A.D. Bobadilla, N.L. Rangel, H. Zhong, M.L. Norton, A. Sinitskii, J.M. Seminario, Nanotechnology 20, 175102 (2009).

[5] T. Kodama, A. Jain, K.E. Goodson, Nanoletters 9, 2005 (2009).

[6] P. Tran, B. Alavi, G. Gruner, Phys. Rev. Lett. 85, 1564 (2000).

[7] H. Boettger, V.V. Bryksin, Hopping Conduction in Solids, Akademie-Verlag, Deerfield Beach, FL 1985.

[8] A. Kaynak, Tr. J. Chem. 22, 81 (1985).

[9] J.G. Simmons, G.W. Taylor, Phys. Rev. B 5, 1619 (1977).

[10] V. Kažukauskas, Semicond. Sci. Technol. 19, 1373 (2004).

[11] V. Kažukauskas, A. Arlauskas, M. Pranaitis, M. Glatthaar, A. Hinsch, J. Nanosci. Nanotechnol. 10, 1376 (2010).

[12] M.K. Sheinkman, A.Y. Shik, Sov. Phys.-Semicond. 10, 128 (1976).

[13] A.M. Nardes, M. Kemerink, R.A.J. Janssen, J.A.M. Bastiaansen, N.M.M. Kiggen, B.M.W. Langeveld, A.J.J.M. van Breemen, M.M. de Kok, Adv. Mater. 19, 1196 (2007).

[14] S. Marciniak, X. Crispin, K. Uvdal, M. Trzcinski, J. Birgerson, L. Groenendaal, F. Louwet, W.R. Salaneck, Synthetic Metals 141, 67 (2004). 